\title{
Isosbestic points: How a narrow crossing region of curves determines their leading parameter dependence
}

\author{
M. Greger, M. Kollar, and D. Vollhardt \\ Theoretical Physics III, Center for Electronic Correlations and Magnetism, Institute of Physics, \\ University of Augsburg, 86135 Augsburg, Germany \\ (Received 21 December 2012; published 29 May 2013)
}

\begin{abstract}
We analyze the sharpness of crossing ("isosbestic") points of a family of curves which are observed in many quantities described by a function $f(x, p)$, where $x$ is a variable (e.g., the frequency) and $p$ is a parameter (e.g., the temperature). We show that if a narrow crossing region is observed near $x_{*}$ for a range of parameters $p$, then $f(x, p)$ can be approximated by a perturbative expression in $p$ for a wide range of $x$. This allows us, e.g., to extract the temperature dependence of several experimentally obtained quantities, such as the Raman response of $\mathrm{HgBa}_{2} \mathrm{CuO}_{4+\delta}$, photoemission spectra of thin $\mathrm{VO}_{2}$ films, and the reflectivity of $\mathrm{CaCu}_{3} \mathrm{Ti}_{4} \mathrm{O}_{12}$, all of which exhibit narrow crossing regions near certain frequencies. We also explain the sharpness of isosbestic points in the optical conductivity of the Falicov-Kimball model and the spectral function of the Hubbard model.
\end{abstract}

DOI: 10.1103/PhysRevB.87.195140

PACS number(s): 71.27.+a, 71.10.Fd

\section{INTRODUCTION}

As early as 1937 it was noticed ${ }^{1}$ that the curves of the extinction coefficient $\alpha\left(\omega, c_{1}\right)$ of a mixture of two liquid solutions intersect exactly at one frequency $\omega^{\star}$ when plotted as a function of $\omega$ for different concentrations $c_{1}$ of one of the components. At this particular frequency, the extinction coefficient therefore no longer depends on the parameter $c_{1}$. This point of intersection of the family of curves $\alpha\left(\omega, c_{1}\right)$ is referred to as an isosbestic point. ${ }^{2-4}$ Today isosbestic points of the extinction coefficient of two compounds play an important role in spectrophotometry. ${ }^{5}$

It is a common practice in the natural sciences to illustrate the functional dependence of a quantity $f$ on a variable $x$ and a parameter $p$ by plotting $f(x, p)$ versus $x$ for several values of $p$, say $p_{1}, \ldots, p_{n}$. This leads to a family of $n$ curves $f\left(x, p_{i}\right)$, $i=1, \ldots, n$. The curves will usually intersect. If the points of intersection are confined to a narrow region of $x$ values, or if they even coincide at a single point $x^{\star}$ as in the case of the absorbance $\alpha\left(\omega, c_{1}\right)$, it leads to a conspicuous feature whose origin calls for an explanation. Well-defined crossing points of curves are now often called "isosbestic points" even if they do not fall onto a single point. ${ }^{6,7}$ We will use this term also in the present paper. We stress that the characteristic $p$-independence of $f(x, p)$ at $x^{\star}$ in these general cases is only fulfilled in a fairly small $p$-interval, i.e., it is a "local" phenomenon.

For example, an unexpected sharp crossing point at $T^{\star}=$ $160 \mathrm{mK}$ was recognized in the family of curves of the specific heat $c_{V}(T)$ of normal-liquid ${ }^{3} \mathrm{He}$ when plotted as a function of temperature for different molar volumes. ${ }^{8}$ Rather sharp crossing points are also observed ${ }^{9}$ in the specificheat curves $c(T, X)$ of many heavy-fermion compounds, with $X$ as a second thermodynamic variable, e.g., when measured at different pressures $(X=P)$ as in $\mathrm{CeAl}_{3},{ }^{10}$ or for different magnetic fields $(X=H)$ as in $\mathrm{CeCu}_{6-x} \mathrm{Al}_{x},{ }^{11}$ $\mathrm{RuSr}_{2} \mathrm{Gd}_{1.5} \mathrm{Ce}_{0.5} \mathrm{Cu}_{2} \mathrm{O}_{10-\delta},{ }^{12} \mathrm{Mn}_{1-x} \mathrm{Fe}_{x} \mathrm{Si}^{13} \mathrm{Mn}_{1-x} \mathrm{Co}_{x} \mathrm{Si}^{13}$ $\mathrm{Cu}_{2} \mathrm{OSeO}_{3},{ }^{14}$ and $\mathrm{MnSi}^{15}$

Crossing points of specific-heat curves have been found in theoretical investigations of lattice models for correlated electrons such as the one-band Hubbard model. ${ }^{9,16-18}$ At half-filling the curves obtained by plotting $c(T, U)$ versus $T$ for different values of the local Coulomb repulsion $U$ always cross at two temperatures, irrespective of the type of lattice, as seen in the case of nearest-neighbor hopping in $d=1,{ }^{19-21} d=2,{ }^{22-25}$ and $d=\infty,{ }^{17}$ as well as for long-range hopping in $d=1 .{ }^{26}$ In particular, the crossing point at high temperatures was found to occur at a nearly universal value $c^{\star} / k_{B} \simeq 0.34 .^{18,27}$ Subsequently, crossing points were noticed also in the charge susceptibility of the half-filled extended Hubbard model in $d=1^{28}$ and the thermal conductance of strongly correlated quantum dots. ${ }^{29}$ The heavy fermion compound $\mathrm{YbRh}_{2} \mathrm{Si}_{2}$ exhibits analogous isosbestic points in its magnetic susceptibility $\chi(H, T){ }^{30}$

The crossing points discussed above all occur when the temperature dependence of some physical quantity such as the specific heat is plotted for different values of a parameter, e.g., pressure, magnetic field, or interaction strength. But crossing points have also been observed in experimental data and theoretical results for the frequency dependence of various response functions, e.g., (i) in the optical conductivity $\sigma(\omega, X)$ of electron-doped $\mathrm{Nd}_{2-\delta} \mathrm{Ce}_{\delta} \mathrm{CuO}_{4}$, with $X=\delta$ as the doping level, ${ }^{6}$ and other high- $T_{c}$ materials ${ }^{31}$ as well as in the pyrochlore-type molybdate family $R_{2} \mathrm{Mo}_{2} \mathrm{O}_{7}(R=$ Nd,Sm,Eu,Gd), ${ }^{32}$ where $X$ now denotes the ionic radius $r$ associated with the different elements $R$; (ii) in the Raman response function $\chi(\omega, T)$ of the high- $T_{c}$ material $\mathrm{HgBa}_{2} \mathrm{CuO}_{4+\delta}$ plotted as a function of the Raman shift $\omega$ for different temperatures; ${ }^{33}$ and (iii) in the dielectric function $\epsilon(\omega, T)$ of the colossal magnetoresistance material $\mathrm{LaMnO}_{3}{ }^{34}$ and the reflectivity $R(\omega, T)$ of the colossal dielectric constant material $\mathrm{CaCu}_{3} \mathrm{Ti}_{4} \mathrm{O}_{12}{ }^{35}$ when plotted as a function of the photon energy $\omega$ for different values of $T$.

Sharp crossing points in the Raman response $\chi(\omega, T)$ have been found in theoretical investigations based on the Hubbard model $^{7,36}$ and the Falicov-Kimball model. ${ }^{37,38}$ The spectral function $A(\omega, U)$ of the Hubbard model computed within dynamical mean-field theory (DMFT) also shows a sharp crossing point. ${ }^{39}$

Whenever isosbestic points occur in a function $f(x, p)$, two separate questions arise: ${ }^{9}$ 
(i) Why do the curves cross at all?

(ii) Why is the crossing feature confined to a narrow region despite significant changes in the parameter $p ?^{40}$

As discussed by one of us, ${ }^{9}$ the existence of crossing points in specific-heat curves can be explained by a sum rule for the change of the entropy $S(T, X)$ with respect to $X$ in the limit of $T \rightarrow \infty$. Furthermore, their sharpness can be linked to the smallness of the susceptibilities $\chi^{(n)}(T, X)=\partial^{n} \xi / \partial X^{n}$ of strongly correlated systems in an expansion of the specific heat $c(T, X)$ with respect to $X$, where $\xi(T, X)$ is the conjugate variable to $X .^{41}$

In this paper, we concentrate on the second question. In Sec. II we discuss the properties of isosbestic points and derive the leading parameter dependence for a function $f(x, p)$ with an approximate isosbestic point. This yields a generalization of the two-fluid model ${ }^{1}$ and allows us to extract the leading parameter dependence for quantities which exhibit such a narrow crossing region. In Sec. III we then extract the temperature dependence of several experimentally obtained quantities, such as the Raman response $\chi(\omega, T)$ of the cuprate compound $\mathrm{HgBa}_{2} \mathrm{CuO}_{4+\delta}$, in photoemission spectra $I(\omega, T)$ of $\mathrm{VO}_{2}$ thin films, and in the reflectivity $R(\omega, T)$ of $\mathrm{CaCu}_{3} \mathrm{Ti}_{4} \mathrm{O}_{12}$. We also explain the sharpness of isosbestic points in the optical conductivity of the FalicovKimball model $^{37}$ (Sec. IV) and the spectral function $A(\omega, U)$ of the Hubbard model (Sec. V), both within DMFT. ${ }^{42-47}$ A conclusion follows in Sec. VI.

\section{PROPERTIES OF EXACT AND APPROXIMATE ISOSBESTIC POINTS}

\section{A. Existence of crossing points}

A family of nonmonotonic curves, obtained by plotting a quantity $f(x, p)$ as a function of $x$ for different values of a parameter $p$, will in general intersect. The crossing points are located along a curve $x^{\star}(p)$ defined by ${ }^{9,39}$

$$
\left.\frac{\partial f(x, p)}{\partial p}\right|_{x^{\star}(p)}=0 .
$$

If these points of intersection are confined to a narrow region (in which case we refer to them as isosbestic points), the value $x^{\star}(p)$ depends only weakly on $p$. If the curves intersect at a single point, $x^{\star}$ does not depend on $p$ at all.

Equation (1) immediately explains why the absorbance of a mixture of two liquid solutions with individual concentrations $c_{1}, c_{2}$, whose total concentration is constant $\left(c_{1}+c_{2}=c\right)$, has a sharp isosbestic point. ${ }^{2}$ Namely, in this case the absorbance $\alpha\left(\omega, c_{1}\right)$ depends only linearly on the concentration $c_{1}$, i.e., it has the special form

$$
\alpha\left(\omega, c_{1}\right)=c_{1} \alpha_{1}(\omega)+\left(c-c_{1}\right) \alpha_{2}(\omega) .
$$

If $\alpha_{1}$ and $\alpha_{2}$ coincide at some frequency $\omega^{\star}$, i.e., $\alpha_{1}\left(\omega^{\star}\right)=$ $\alpha_{2}\left(\omega^{\star}\right)$, then

$$
\left.\frac{\partial \alpha\left(\omega, c_{1}\right)}{\partial c_{1}}\right|_{\omega^{\star}}=0 .
$$

This implies that for all concentrations $c_{1}$ the absorbance curves intersect exactly at one frequency $\omega^{\star}$, or the equivalent wavelength. Quite generally, whenever a system is a superposition of two components, where the sum of the densities is conserved, isosbestic points in the curves plotted for different densities are bound to occur. ${ }^{39,48}$ Indeed, this argument is used by Uchida et al. ${ }^{6}$ and Kézsmárki et al..$^{32}$ to explain the well-defined isosbestic points in the optical conductivity $\sigma(\omega, p)$ of numerous correlated electron materials, where $p$ is some control parameter, e.g., the temperature or the bandwidth. Starting from the sum rule for the optical conductivity, and assuming (i) that the spectral weight is only redistributed between electrons with two different energy scales, and (ii) the optical spectrum can be decomposed and linearly interpolated by two terms as a function of $p$, they arrive at an expression for the optical conductivity given by $\sigma(\omega, p)=p \sigma_{1}(\omega)+(1-p) \sigma_{2}(\omega)$, which is identical to the result for the absorbance in Eq. (2) and hence leads to an isosbestic point at some particular frequency $\omega^{\star} .{ }^{49}$

Before we continue with our discussion, we need to introduce a suitable nomenclature. Based on the behavior of the curve of crossing points $x^{\star}(p)$, we identify three different cases: (i) A globally exact isosbestic point, which is characterized by $x^{\star}(p)=$ const, corresponding to a complete $p$ independence of $f\left(x^{\star}, p\right)$; (ii) a locally exact isosbestic point, which arises when $x^{\star}(p)$ exhibits a local extremum or a higher-order stationary point around some value $p=$ $p_{0}$, corresponding to a locally $p$-independent $f\left(x^{\star}(p), p\right)$ around $p=p_{0}$; (iii) an approximate isosbestic point, which corresponds to a weak $p$ dependence of $x^{\star}(p)$.

We note that locally exact isosbestic points can be further classified by the order of the stationary point of $x^{\star}(p)$ at $p_{0}$. If

$$
\left.\frac{\partial^{k} x^{\star}(p)}{\partial p^{k}}\right|_{p_{0}}=0
$$

for $k \leqslant n$, the isosbestic point is called an "isosbestic point of $n$th order."

\section{B. Sharpness of isosbestic points}

To understand the general origin of sharp isosbestic points in a function $f(x, p)$, it is important to note that isosbestic behavior is usually observed only in a certain parameter range around some particular value $p_{0}$ and can be expected to break down away from $p_{0}$. Accordingly, the weak $p$ dependence of $x^{\star}(p)$ required for an approximate isosbestic point will generally be a local phenomenon, i.e., it applies only for a finite $p$ interval around $p_{0}$. This observation of the local nature of isosbestic points motivates the following expansion around $p_{0}$ :

$$
f(x, p)=f\left(x, p_{0}\right)+\left(p-p_{0}\right) F_{1}\left(x, p_{0}\right)+O\left[\left(p-p_{0}\right)^{2}\right],
$$

where $F_{n}(x, p)=\partial^{n} f(x, p) / \partial p^{n}$. One has $F_{1}\left(x^{\star}\left(p_{0}\right), p_{0}\right)=0$, by virtue of the definition of $x^{\star}(p)$ [Eq. (1)]. As a consequence, the linear approximation of $f(x, p)$,

$$
f_{\text {approx }}(x, p)=f\left(x, p_{0}\right)+\left(p-p_{0}\right) F_{1}\left(x, p_{0}\right),
$$

will exhibit an exact isosbestic point at $x=x^{\star}\left(p_{0}\right)$ for all values of $p$. Since

$$
f(x, p)=f_{\text {approx }}(x, p)+O\left[\left(p-p_{0}\right)^{2}\right],
$$


the second term is seen to be responsible for deviations from the exact isosbestic point described by $f_{\text {approx }}(x, p)$. This result can be put to a test by checking the validity of the following approximation:

$$
\begin{aligned}
\tilde{f}(x, p) & \equiv f(x, p)-\left(p-p_{0}\right) F_{1}\left(x, p_{0}\right) \\
& =f\left(x, p_{0}\right)+O\left[\left(p-p_{0}\right)^{2}\right] \\
& \simeq f\left(x, p_{0}\right) .
\end{aligned}
$$

The quantity $\tilde{f}(x, p)$ represents the reference curve $f\left(x, p_{0}\right)$ plus all terms which are responsible for the deviations from the exact isosbestic case. The verification of its approximate parameter independence, i.e., the verification of the approximation $\tilde{f}(x, p) \simeq f\left(x, p_{0}\right)$, allows one to quantify the importance of the subleading terms $O\left[\left(p-p_{0}\right)^{2}\right]$ and therefore the deviations from the exact isosbestic case.

In general, the value of $p_{0}$ should correspond to the position of the weakest $p$ dependence of $x^{\star}(p)$ and, without any further information on the system, is generally hard to determine unambiguously. Of particular interest, however, are the cases in which $p_{0}$ corresponds to an extremum or some higher-order stationary point of $x^{\star}(p)$. These cases lead to locally exact isosbestic points where $p_{0}$ is determined by $x^{\star^{\prime}}\left(p_{0}\right)=0$. The new variable $\left(p-p_{0}\right)$ is then a small parameter of the system, e.g., an internal frequency scale if $p$ is a frequency. From the implicit Eq. (1) one obtains

$$
\frac{d}{d p} x^{\star}(p)=-\left.\frac{\partial F_{1}(x, p) / \partial p}{\partial F_{1}(x, p) / \partial x}\right|_{x=x^{\star}(p)},
$$

which means that for $x^{\star^{\prime}}\left(p_{0}\right)=0$ the subleading terms in Eq. (7) are at least of order $\left(p-p_{0}\right)^{3}$, thus corresponding to particularly weak deviations from the exact isosbestic point in $f_{\text {approx }}(x, p)$ in a vicinity of $p_{0}$. In practice, isosbestic points corresponding to an extremum of $x^{\star}(p)$ are rather common, as will be discussed in the following sections. A more mathematical treatment regarding the behavior around such stationary points of $x^{\star}(p)$ is provided in Appendix A. In the rest of the paper, we will use these results to show that isosbestic points are indeed connected to a leading parameter dependence as described by Eq. (6).

Finally, we emphasize that it is not possible to quantify the sharpness of isosbestic points beyond Eqs. (8)-(10), since the perceived sharpness always depends on the magnification of the crossing region, which is not an intrinsic property of $f(x, p)$ itself (see Appendix B).

\section{ANALYSIS OF EXPERIMENTALLY OBSERVED ISOSBESTIC POINTS}

The generality of Eq. (7) allows us to investigate the sharpness of a whole class of isosbestic points in entirely unrelated systems within a common framework. An existing set of theoretical or experimental data of a quantity $Q\left(x, p_{i}\right)$ obtained for a number of different parameters, $i=1,2, \ldots, n$ can be analyzed in the vicinity of an isosbestic point $x^{\star}(p)$ by comparing it to

$$
Q_{\text {approx }}(x, p)=Q\left(x, p_{0}\right)+\left(p-p_{0}\right) Q_{1}\left(x, p_{0}\right) .
$$

Here the function $Q_{1}\left(x, p_{0}\right)$ is approximated as

$$
Q_{1}\left(x, p_{0}\right) \simeq \frac{Q\left(x, p_{\alpha}\right)-Q\left(x, p_{\beta}\right)}{\left(p_{\alpha}-p_{0}\right)-\left(p_{\beta}-p_{0}\right)},
$$

with $p_{\alpha}, p_{\beta} \in\left[p_{1}, \ldots, p_{n}\right]$ sufficiently close to $p_{0}$ to obtain a proper approximation of $Q_{1}\left(x, p_{0}\right)$. As before, the applicability of Eq. (12) is then tested by verifying the approximate $p$ independence of

$$
\begin{aligned}
\widetilde{Q}\left(x, p_{i}\right) & =Q\left(x, p_{i}\right)-\left(p-p_{0}\right) Q_{1}\left(x, p_{0}\right) \\
& \simeq Q\left(x, p_{0}\right) .
\end{aligned}
$$

\section{A. Raman response of $\mathrm{HgBa}_{2} \mathrm{CuO}_{4+\delta}$}

We now apply the scheme to the isosbestic point observed in measurements ${ }^{33}$ of the antinodal $\left(\boldsymbol{B}_{1 g}\right)$ Raman response $\chi(\omega, T)$ of the cuprate compound $\mathrm{HgBa}_{2} \mathrm{CuO}_{4+\delta}$ for various temperatures at optimal doping $(\delta=0.16)$, which shows a distinct isosbestic point for up to $\simeq 100 \mathrm{~K}$. Proceeding as described above, we make the following Sommerfeld-type ansatz for the low-temperature dependence of $\chi(\omega, T)$ :

$$
\chi(\omega, T)=\chi(\omega, 0)+T^{2} \chi_{2}(\omega)+O\left[T^{3}\right],
$$

where

$$
\chi_{2}(\omega) \simeq \frac{\chi\left(\omega, T_{1}\right)-\chi\left(\omega, T_{2}\right)}{T_{1}^{2}-T_{2}^{2}}
$$

as in Eq. (13). Again, the validity of Eq. (16) can be tested via the $T$ independence of the quantity

$$
\tilde{\chi}(\omega, T)=\chi(\omega, T)-T^{2} \chi_{2}(\omega) \simeq \chi(\omega, T=0) .
$$

This is done in Fig. 1, which shows good agreement with Eq. (18). To determine $\chi_{2}(\omega)$ we chose $T_{1}=14 \mathrm{~K}$ and $T_{2}=$ $90 \mathrm{~K}$, which is by no means a unique combination. ${ }^{50}$

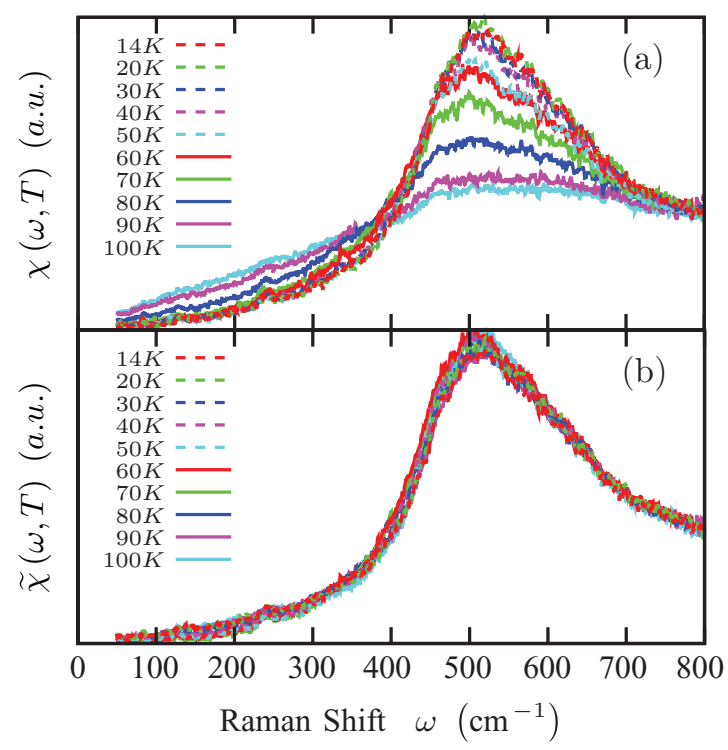

FIG. 1. (Color online) (a) Antinodal Raman response $\chi(\omega, T)$ of $\mathrm{HgBa}_{2} \mathrm{CuO}_{4+\delta}{ }^{33}$ at optimal doping and (b) the function $\tilde{\chi}(\omega, T)$. The weak temperature dependence of $\tilde{\chi}(\omega, T)$ explains the sharpness of the isosbestic point and also confirms the validity of ansatz (16) within the available data. 


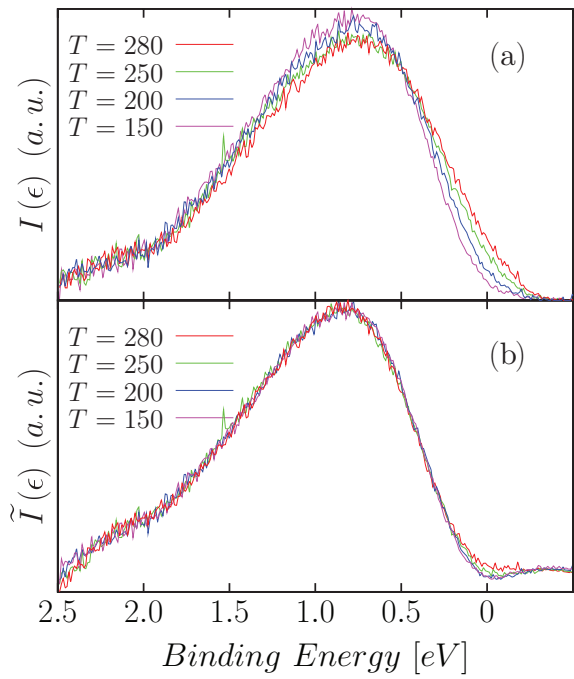

FIG. 2. (Color online) Temperature dependence of (a) photoemission spectra $I(\omega, T)$ of a $\mathrm{VO}_{2}$ thin film ${ }^{51}$ and (b) the function $\widetilde{I}(\omega, T)$ defined in Eq. (20). The weak temperature dependence of $\widetilde{I}(\omega, T)$ explains the sharpness of the isosbestic point and also supports the importance of electron-phonon interaction.

It is interesting to note that the isosbestic point and thus the quadratic $T$ dependence in Eq. (16) are specific features of the optimally doped system and are absent for other dopings ${ }^{33}$ (at least for the provided temperatures). For the case of optimal doping, we have explained the sharp isosbestic point in $\chi(\omega, T)$ by its essentially quadratic temperature dependence.

\section{B. Photoemission spectra of $\mathrm{VO}_{2} / \mathrm{TiO}_{2}(001)$ thin films}

Next we apply the method to explain photoemission spectra $I(\omega, T)$ of $\mathrm{VO}_{2} / \mathrm{TiO}_{2}$ thin films, ${ }^{51}$ which exhibit a marked isosbestic (see Fig. 2). The strong temperature dependence of $I(\omega, T)$ was seen ${ }^{51}$ as an indication of strong electron-phonon coupling. Consequently, $I(\omega, T)$ was $^{51}$ convincingly reproduced through the spectral function $A(\omega, T)$ of the independent boson model through $I(\omega, T) \propto A(\omega, T)$. The temperature dependence of $A(\omega, T)$ with $A(\omega, T)=$ $A(\omega, 0)+T A_{1}(\omega)+O\left(T^{2}\right)$ justifies the following ansatz:

$$
I(\omega, T)=I(\omega, 0)+T I_{1}(\omega)+O\left[T^{2}\right],
$$

where

$$
I_{1}(\omega) \simeq \frac{I\left(\omega, T_{1}\right)-I\left(\omega, T_{2}\right)}{T_{1}-T_{2}}
$$

is extracted from the data $\left(T_{1}=150 \mathrm{~K}\right.$ and $\left.T_{2}=200 \mathrm{~K}\right)$. The ansatz (19) is confirmed by the relative $T$ independence (well within measurement accuracy) of the quantity

$$
\widetilde{I}(\omega, T)=I(\omega, T)-T I_{1}(\omega) \simeq I(\omega, T=0),
$$

plotted in Fig. 2(b). This provides an explanation for the sharpness of the isosbestic point, and the leading linear temperature dependence supports the importance of bosonic excitations along the lines of Ref. 51.

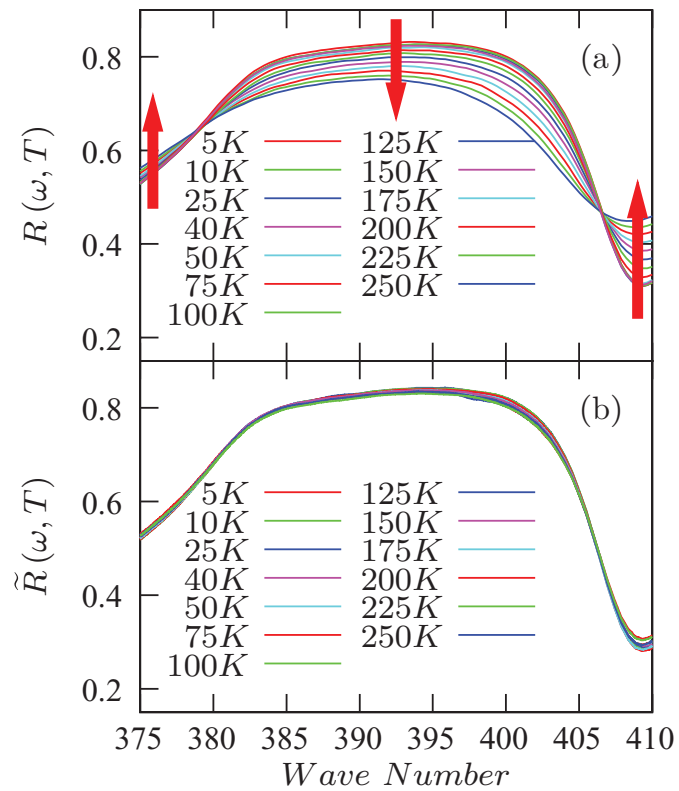

FIG. 3. (Color online) Temperature dependence of (a) the reflectivity $R(\omega, T)$ of $\mathrm{CaCu}_{3} \mathrm{Ti}_{4} \mathrm{O}_{12}{ }^{35}$ and (b) the function $\widetilde{R}(\omega, T)$. The weak temperature dependence of $\widetilde{R}(\omega, T)$ explains the origin of the isosbestic point.

\section{Phonon modes in $\mathrm{CaCu}_{3} \mathrm{Ti}_{4} \mathrm{O}_{12}$}

We investigate isosbestic points in reflectivity $R(\omega, T)$ measurements of $\mathrm{CaCu}_{3} \mathrm{Ti}_{4} \mathrm{O}_{12}$ around phononic excitations. ${ }^{35}$ Again, assuming the leading $T$ dependence to be linear, we proceed along the lines of the preceding section and introduce

$$
\widetilde{R}(\omega, T)=R(\omega, T)-T R_{1}(\omega),
$$

where

$$
R_{1}(\omega) \simeq \frac{R\left(\omega, T_{1}\right)-R\left(\omega, T_{2}\right)}{T_{1}-T_{2}}
$$

is extracted directly from data. Note that the sensitivity of Eq. (21) on the quality of the available data required the use of a comparably large temperature difference $\left(T_{1}=40 \mathrm{~K}, T_{2}=\right.$ $175 \mathrm{~K})$. The high quality of the match in Fig. 3 explains the sharpness of the isosbestic point and also shows that a linear temperature dependence already provides a proper description of the available measurement data.

\section{Isosbestic points in conductive polymers}

Here we analyze the marked isosbestic points in the optical conductivity of conductive polymers ${ }^{52,53}$ as observed in many spectroelectrochemical experiments for different doping levels. By applying an external bias voltage $V$, these experiments allow for direct control of the doping level of the sample and yield the optical conductivity (absorbance) $\sigma(\omega, n)$ $[A(\omega, n)]$ as a function of doping $n$.

To demonstrate the general applicability of our scheme, we specifically consider a subset of the spectroelectrochemical measurements on poly(3,4-ethylenedioxythiophene) (PEDOT) presented in Ref. 54. Note that we consider here $A(\omega, V)$ instead of $A(\omega, n)$ since $V$ is the original parameter in the measurement. We make the following linear ansatz 


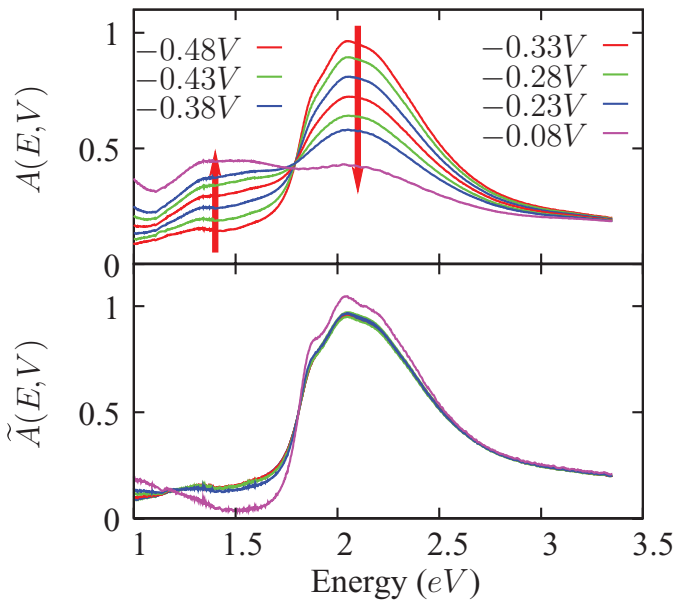

FIG. 4. (Color online) The upper plot shows the marked isosbestic point in spectroelectrochemical experiments for PEDOT. In the lower plot we show the corresponding curves $\widetilde{A}\left(\omega, V_{i}\right)$, which (except for $V_{7}=-0.08 \mathrm{~V}$ ) essentially all collapse onto a single curve. We included the curves $A\left(\omega, V_{7}\right)$ and $\widetilde{A}\left(\omega, V_{7}\right)$ to demonstrate that deviations become important at more extreme parameter values. In particular, we note that $A\left(\omega, V_{7}\right)$ does not contribute to the isosbestic defined by the other curves (Data from Ref. 54.).

for the parameter dependence of $A(\omega, V)$, expanding around $V_{0}=-0.48 \mathrm{~V}$ :

$$
A(\omega, V)=A\left(\omega, V_{0}\right)+\left(V-V_{0}\right) A_{1}(\omega),
$$

where

$$
A_{1}(\omega) \simeq \frac{A\left(\omega, V_{1}\right)-A\left(\omega, V_{2}\right)}{V_{1}-V_{2}}
$$

with $V_{1}=-0.48$ and $V_{2}=-0.38$. Again we plot both $A(\omega, V)$ and $\widetilde{A}(\omega, V) \equiv A(\omega, V)-\left(V-V_{0}\right) A_{1}(\omega)$ in Fig. 4, verifying the applicability of our general scheme and in particular of Eq. (22). Our analysis thus reveals that the seemingly complicated behavior of $A(E, V)$ in Fig. 4 is in fact due to a linear voltage dependence.

\section{E. Isosbestic point in the dielectric function of $\mathrm{LaMnO}_{3}$}

Here we address the conspicuous isosbestic points in the dielectric function $\epsilon_{1}(\omega, T)$ of $\mathrm{LaMnO}_{3}$ for different temperatures $^{55}$ (see Fig. 5). We apply the same analysis as before, i.e., we use the following Sommerfeld-type ansatz for the temperature dependence of $\epsilon_{1}(\omega, T)$ :

$$
\epsilon_{1}(\omega, T)=\epsilon_{1}(\omega, 0)+T^{2} \epsilon_{1}^{(1)}(\omega)+O\left[T^{3}\right],
$$

where $\left(T_{1}=20 \mathrm{~K}, T_{2}=90 \mathrm{~K}\right)$

$$
\epsilon_{1}^{(1)}(\omega) \simeq \frac{\epsilon_{1}\left(\omega, T_{1}\right)-\epsilon_{1}\left(\omega, T_{2}\right)}{T_{1}^{2}-T_{2}^{2}}
$$

as in Eq. (13). Again, the validity of Eq. (16) is tested via the $T$ independence of the quantity

$$
\widetilde{\epsilon}_{1}(\omega, T)=\epsilon_{1}(\omega, T)-T^{2} \epsilon_{1}^{(1)}(\omega) .
$$

This reveals (i) that the origin of the optical response is dominated by electronic excitations that are quadratic in $T$, and (ii) that the temperatures are still moderately small as compared to the electronic low-energy scales of the system.

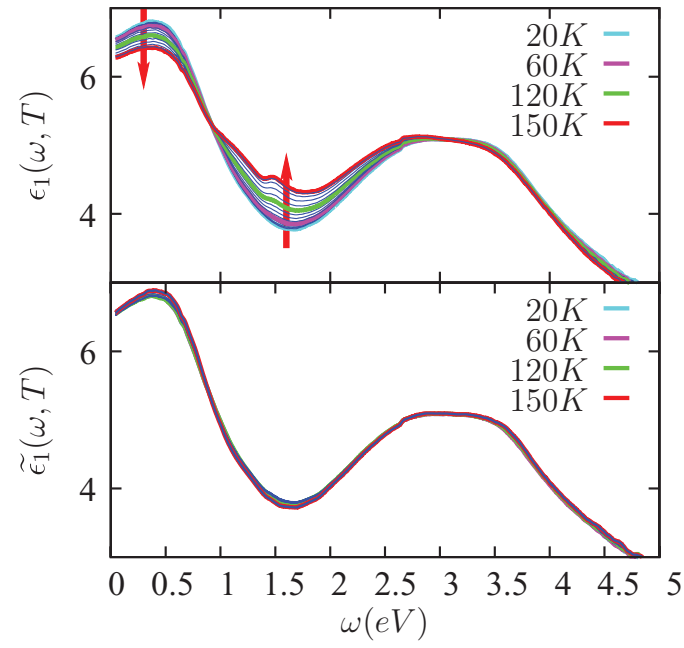

FIG. 5. (Color online) Temperature variation of the real part of the dielectric function spectra $\epsilon_{1}(\omega, T)$ of $\mathrm{LaMnO}_{3}$ between 20 and $140 \mathrm{~K}$ in $10 \mathrm{~K}$ increments (Data from Ref. 55.).

\section{ISOSBESTIC POINTS IN THE OPTICAL CONDUCTIVITY OF THE FALICOV-KIMBALL MODEL}

\section{A. Model and optical conductivity in DMFT}

The Falicov-Kimball model ${ }^{37,56}$ describes itinerant electrons ( $c$ electrons) interacting with localized electrons $(f$ electrons) by a local Coulomb repulsion. The Hamiltonian is given by

$$
\begin{aligned}
H= & -\sum_{i, j} t_{i, j} c_{i}^{\dagger} c_{j}-\mu \sum_{i}\left(c_{i}^{\dagger} c_{i}+f_{i}^{\dagger} f_{i}\right)+E_{f} \sum_{i} f_{i}^{\dagger} f_{i} \\
& +U \sum_{i}\left[f_{i}^{\dagger} f_{i}-\frac{1}{2}\right]\left[c_{i}^{\dagger} c_{i}-\frac{1}{2}\right] .
\end{aligned}
$$

It can be viewed as a Hubbard model with spin-dependent hopping and two different chemical potentials. The symbols $c_{i}^{\dagger}\left(f_{i}^{\dagger}\right)$ and $c_{i}\left(f_{i}\right)$ denote the itinerant (localized) electron creation and annihilation operators. The chemical potential $\mu$ constrains the total number of $c$ electrons, while $E_{f}$ is an orbital energy of the localized electrons. Here $U$ is an on-site repulsion between the two electron species.

At low temperatures, the optical conductivity of the FalicovKimball model with the Gaussian density of states (DOS) is known to exhibit a distinct isosbestic point ${ }^{37}$ when plotted for various $c$-electron densities (Fig. 6). In the following, we will discuss the existence and sharpness of isosbestic points and concentrate on the ungapped system at zero temperature and use the semielliptic DOS $\rho(\epsilon)=(2 \pi t)^{-1} \sqrt{4 t^{2}-\epsilon^{2}}$ with $t=1$.

Within dynamical mean-field theory (DMFT), the model can be solved exactly for the Green function $G(\omega)$ or the self-energy $\Sigma(\omega)$ due to the simplicity of the impurity problem: ${ }^{57}$

$$
G(\omega)=\frac{1-w_{1}}{\mathcal{G}_{0, c}^{-1}(\omega)}+\frac{w_{1}}{\mathcal{G}_{0, c}^{-1}(\omega)-U},
$$

where $\mathcal{G}_{0, c}^{-1}(\omega)=\Sigma(\omega)+G^{-1}(\omega)$ denotes the Green function of the effective medium (Weiss field). The weight $w_{1}$ equals the average $f$-electron concentration, which will be set to $1 / 2$ 


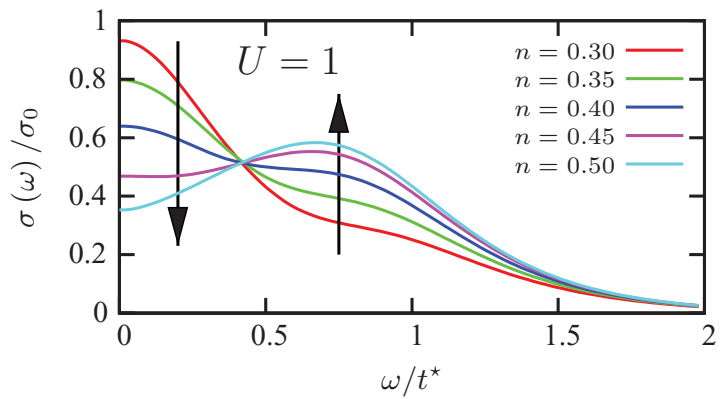

FIG. 6. (Color online) Isosbestic point in the optical conductivity of the Falicov-Kimball model at $T=0^{37}$ (Gaussian DOS $\left[U_{c} \simeq 1.5\right.$ ] with density $n$ of mobile and density $w_{1}=1 / 2$ of immobile particles). Spectral weight is transferred from low frequencies to the Hubbard peak as electron density is increased, producing an isosbestic point at medium frequencies.

in the following. Due to particle-hole symmetry, it is sufficient to restrict the treatment to $c$-electron densities $n \leqslant 1 / 2$, i.e., $\mu \leqslant 0$.

The optical conductivity in DMFT is essentially given by the particle-hole bubble ${ }^{58}$ which on the real axis corresponds to

$$
\begin{aligned}
& \sigma(\omega)= \sigma_{0} \int_{-\infty}^{\infty} d \epsilon \rho(\epsilon) v(\epsilon)^{2} \int d \omega^{\prime} A_{\epsilon}\left(\omega^{\prime}\right) A_{\epsilon}\left(\omega^{\prime}+\omega\right) \\
& \times \frac{f\left(\omega^{\prime}\right)-f\left(\omega^{\prime}+\omega\right)}{\omega} \\
& A_{\epsilon}(\omega)=-1 / \pi \operatorname{Im}[\omega+\mu-\Sigma(\omega)-\epsilon]^{-1}
\end{aligned}
$$

where $A_{\epsilon}(\omega)$ denotes the spectral density and $\rho(\epsilon)$ the DOS. We fix the velocity function $v(\epsilon)$ according to the treatment in Ref. 59.

\section{B. Existence of isosbestic points in $\sigma(\omega)$}

The formation of isosbestic points in the optical conductivity $\sigma(\omega)$ when plotted for different doping levels of the $c$ electrons in Fig. 6 can easily be deduced on physical grounds, viewing the Falicov-Kimball model as a disordered medium in which spinless electrons interact with localized impurities. In this picture, the scattering rate $\tau^{-1}(\mu) \propto-\operatorname{Im} \Sigma_{\mu}(\omega=0)$ is monotonically related to the $c$-electron density through the chemical potential $\mu$, and reaches a maximum at half-filling [see Fig. 7(b)]. Since $\tau^{-1}(0)$ diverges at the metal-to-insulator transition, it is always possible to suppress the dc conductivity of the half-filled FK model simply by increasing $U$. Thus, in the intermediate $U$ regime, $\sigma_{\mathrm{dc}}(U, n)$ will have a minimum or a $\operatorname{dip}^{37}$ around half-filling, which means $\partial \sigma_{\mathrm{dc}} / \partial n<0$ for $n<1 / 2$ [see Fig. 7(a)]. Besides the mathematical reasoning in Appendix D, a positive sign of $\partial \sigma(\omega, n) / \partial n$ for nonzero frequencies and for sufficiently strong $U$ can be established by taking into account the formation of the well-known charge-transfer or Hubbard peak at $\omega_{C}(U, n) \simeq U$ (see Fig. 6), which is a property of the optical conductivity of correlated systems. Representing a robust indicator of the increasing correlation between $f$ and $c$ electrons, the peak becomes increasingly pronounced as the system is doped toward halffilling. For sufficiently strong $U$ we thus have $\partial \sigma\left(\omega_{C}\right) / \partial n>$

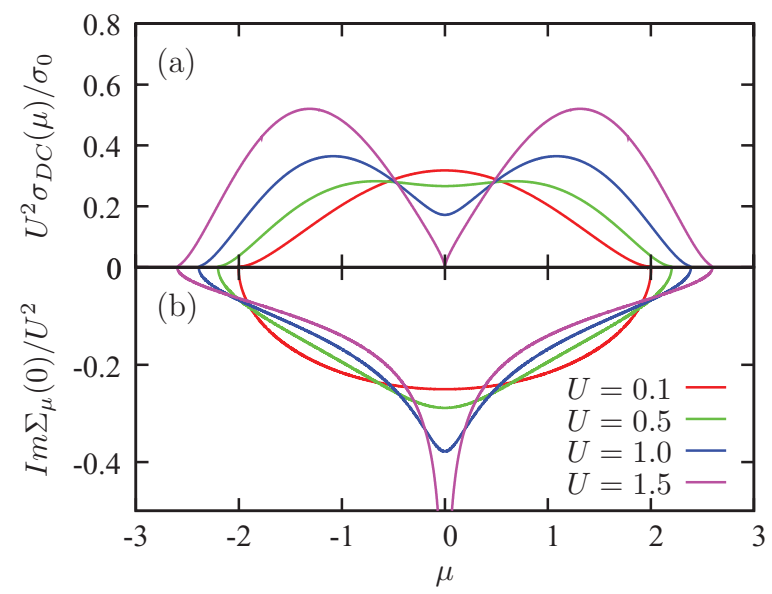

FIG. 7. (Color online) Doping dependence of (a) the rescaled dc conductivities $U^{2} \sigma_{\mathrm{dc}}(\mu)$ and (b) self-energies $\operatorname{Im} \Sigma_{\mu}(0) / U^{2}$ for the semielliptic DOS. With growing interaction, $\operatorname{Im} \Sigma_{\mu}(0)$ becomes increasingly peaked with an extremum at half-filling $(\mu=0)$. Therefore, the scattering rate $\tau^{-1} \propto-\operatorname{Im} \Sigma(0)$ becomes maximal at half-filling. The metal-insulator transition at $U=2$ is distinguished by a vanishing dc conductivity and a diverging scattering rate $\tau^{-1}$. For sufficiently high yet finite $U$, this generic property of the FK model leads to the formation of minima in $\sigma_{\mathrm{dc}}(\mu)$ for $\mu=0$. This corresponds to $\partial \sigma_{\mathrm{dc}}(\mu) / \partial \mu<0$. Note that there exists a one-to-one mapping between $\mu$ and the carrier density $n$. In Appendix E, we show that $\Sigma_{\mu}(\omega)=\Sigma(\omega+\mu)$.

0 and $\partial \sigma_{\mathrm{dc}} / \partial n<0$, and as a consequence there exists a crossing point $\omega^{\star}(n, U)$ [cf. Eq. (1)] with $0<\omega^{\star}<\omega_{C}$ and $\partial \sigma\left(\omega^{\star}, n\right) / \partial n=0$ as depicted in Fig. 6. The formation of isosbestic points is thus an effect of the correlation associated transfer of spectral weight from low to higher frequencies as the system is doped toward half-filling. The argument does not depend on the specifics of the density of states under consideration and thus applies to Fig. 6 (Gaussian DOS) as well as to Fig. 8 (semielliptic DOS).

\section{Sharpness of isosbestic points in $\sigma(\omega)$}

The symmetric density dependence of the crossing frequency $\omega^{\star}(n)$ depicted in Fig. 9 reflects the important role of particle-hole symmetry for locally exact isosbestic points in $\sigma(\omega, n)$. Because of the existence of the one-to-one correspondence between the chemical potential $\mu$ and the carrier density $n$ in the ungapped system, both parameters are equivalent. The chemical potential, however, represents the more convenient parameter because of the simple $\mu$ dependence of the self-energy $\Sigma(\omega)$ (see Appendix E) and of the spectral densities $A_{\epsilon}(\omega)$ in Eq. (27). Thus, concentrating on ungapped systems, we expand around half-filling, i.e., $\mu=0$, taking $\mu$ as a small parameter,

$$
\sigma(\omega, \mu)=\sigma(\omega, 0)+\frac{1}{2} \mu^{2} \frac{\partial^{2}}{\partial \mu^{2}} \sigma(\omega, \mu=0)+O\left(\mu^{4}\right) .
$$

With Eq. (7) we are directly led to the conclusion that the first two terms constitute an exact isosbestic point, while corrections of order $O\left(\mu^{4}\right)$ introduce the $\mu$ dependence into $\omega^{\star}(\mu)$ [or $\omega^{\star}(n)$ ]. According to Eq. (8), we therefore 


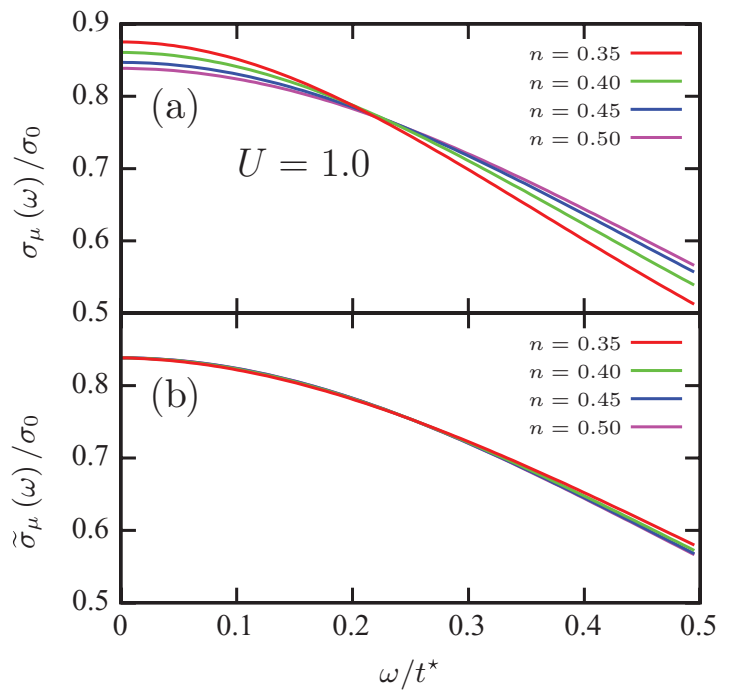

FIG. 8. (Color online) Optical conductivity (a) $\sigma(\omega, \mu(n))$ and (b) the quantity $\widetilde{\sigma}(\omega, \mu(n))$ for the Falicov-Kimball model with the semielliptic DOS. The curves $\widetilde{\sigma}(\omega, \mu(n))$ in (b) essentially lie on top of $\sigma(\omega, \mu=)$ for the substantial density interval $n \in[0.35, \ldots, 0.5]$. Thus, the parameter dependence in $\sigma(\omega, \mu(n))$ essentially stems from the first contribution, which is accompanied by an isosbestic point. The second derivative of $\sigma(\omega, \mu)$ with respect to $\mu$, which is needed for the calculation of $\widetilde{\sigma}(\omega, \mu)$, is obtained from the exact solution. ${ }^{60}$

introduce

$$
\begin{aligned}
\widetilde{\sigma}(\omega, \mu) & =\sigma(\omega, \mu)-\frac{1}{2} \mu^{2} \frac{\partial^{2}}{\partial \mu^{2}} \sigma(\omega, \mu=0) \\
& =\sigma(\omega, \mu=0)+O\left[\mu^{4}\right]
\end{aligned}
$$

and test its approximate $\mu$ independence in a vicinity of $\omega^{\star}(\mu=0)$. This is confirmed graphically in Fig. 8; the obvious agreement with the reference curve can be seen as a measure of the weak influence of higher-order contributions. This provides an especially simple and meaningful interpretation of the approximate isosbestic points in $\sigma(\omega, n)$ : Namely, plots of the conductivity showing this behavior exhibit a density dependence which can be treated in perturbation theory for the parameters under consideration.

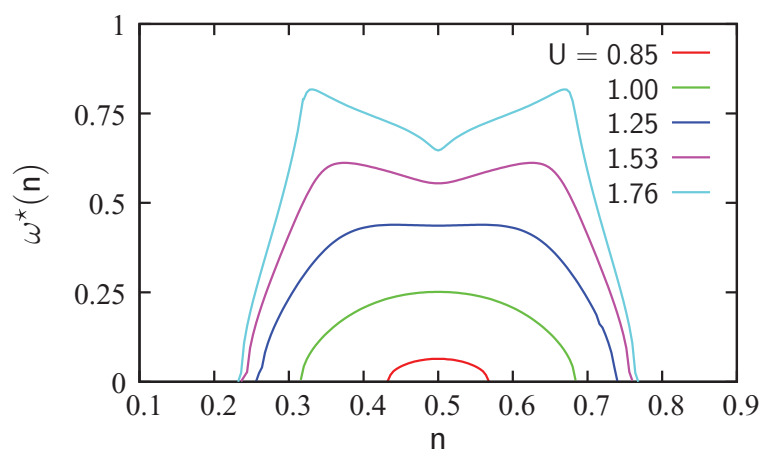

FIG. 9. (Color online) Isosbestic points $\omega^{\star}(n, U)$ of the ungapped system using the semielliptic density of states. The symmetry around half-filling is a consequence of particle-hole symmetry.

\section{ISOSBESTIC POINTS IN THE SPECTRAL FUNCTION OF THE HUBBARD MODEL}

We now apply our approach to the prominent isosbestic point in the spectral function $A(\omega, U)$ of the particle-hole symmetric Hubbard model within DMFT for which, employing symmetry arguments, the crossing frequency was shown ${ }^{39}$ to obey the following dependence on $U$ :

$$
\omega^{\star}(U)=c_{0}+c_{1} U^{2}+O\left[U^{3}\right] .
$$

For a symmetric DOS with van Hove singularities at the band edges $($ at $\pm b$ ) like the semielliptic DOS, Eq. (30) takes the more specific form $\omega^{\star}(U)=b+c_{1} U^{2}+O\left(U^{3}\right)$.

From the infinite slope of the spectral function $A(\omega, 0)$ at $\omega=\omega^{\star}(0)$, it then follows that, in the limit $U \rightarrow 0$, one can never obtain a sharp crossing point defined by the coordinates $(x(U), y(U))$ with $x(U)=\omega^{\star}(U)$ and $y(U)=A\left(\omega^{\star}(U), U\right)$. To avoid this additional mathematical complication, let us consider here the Gaussian DOS with infinite bandwidth, since this DOS is a smooth function so that a weak parameter dependence of $\omega^{\star}(U)$ always translates to a sharp isosbestic point. From particle-hole symmetry, we have the following perturbative expansion:

$$
A(\omega, U)=\sum_{n \geqslant 0} U^{2 n} A_{2 n}(\omega)
$$

with

$$
A_{2}(\omega)=-\frac{1}{\pi} \operatorname{Im}\left\{\int d \epsilon \rho(\epsilon) \frac{\Sigma_{2}(\omega)}{\left[\omega+i 0^{+}-\epsilon\right]^{2}}\right\} .
$$

Here $\Sigma_{2}(\omega)$ denotes the self-energy in second-order perturbation theory ${ }^{61}$ in $U$. We thus obtain from Eqs. (8)-(10)

$$
\widetilde{A}(\omega, U)=A(\omega, U)-U^{2} A_{2}(\omega)
$$

and again obtain $\widetilde{A}(\omega, U) \simeq A(\omega, 0)$, as can be seen from Fig. 10.

\section{CONCLUSION}

In this paper, we developed a framework which allows one to extract information about a correlated system by analyzing the sharpness of an approximate isosbestic point in a physical quantity $f(x, p)$. Our central result is given by Eq. (7), which corresponds to a straightforward generalization of Eq. (2), describing exact isosbestic points. ${ }^{1,2}$ We established a direct connection between the sharpness of isosbestic points and the existence of a small parameter $\delta_{p}=p-p_{0}$, which can be used to extract the leading parameter dependence of the system in the parameter interval under consideration.

For example, our analysis of the Raman response of $\mathrm{HgBa}_{2} \mathrm{CuO}_{4+\delta}$ showed that it has a quadratic temperature dependence only for optimal doping, which raises the question of whether other dynamical quantities exhibit such behavior as well. Another interesting question is whether other (optimally doped) cuprate compounds have similar isosbestic points in their Raman spectra. Furthermore, we deduced a nonobvious linear voltage dependence for the absorbance of the conductive polymer PEDOT in a wide frequency range.

In summary, if a sharp isosbestic point is found in experimental data, an analysis as in Secs. III A-III E should be 


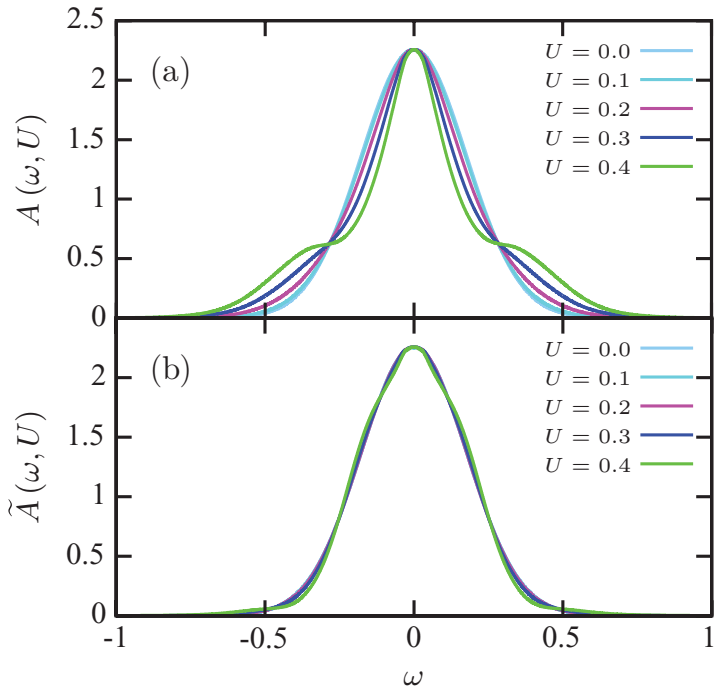

FIG. 10. (Color online) Numerical renormalization group calculation $^{62,63}$ of the spectral function for the half-filled Hubbard model (a) using the Gaussian DOS $\rho(\epsilon)=4 / \sqrt{\pi} \exp \left[-(4 \epsilon)^{2}\right]$. The quantity $\widetilde{A}(\omega, U)$ shows practically no $U$ dependency up to $U_{1} \simeq$ 0.35 (b). The deviations from the locally exact isosbestic point at higher $U$ stem from higher order perturbations and become noticeable only above $U_{1}$.

performed to extract the leading parameter dependence of the family of curves.

\section{ACKNOWLEDGMENTS}

We are thankful to J. Deisenhofer, I. Kézsmárki, A. Loidl, and Y. Tokura for useful communication. This work was supported by the Deutsche Forschungsgemeinschaft through TRR 80.

\section{APPENDIX A: SIZE OF THE CROSSING REGION AROUND LOCALLY EXACT ISOSBESTIC POINTS}

We quantify the influence of the higher-order contributions, i.e., the terms responsible for the deviations from $x^{\star}(p)=$ const. For this we investigate the $p$ dependence of an isosbestic point at $\left(x^{\star}(p), p\right)$, with $x^{\star}(p)$ given implicitly by Eq. (1). We will estimate the size of the region where the curves of $f(x, p)$ cross, e.g., for

$$
\begin{aligned}
& x=x^{\star}\left(p_{0}\right)+\delta x, \\
& p=p_{0}+\delta p,
\end{aligned}
$$

in the vicinity of a certain parameter value $p_{0}$.

We first approximate $f(x, p)$ near $p_{0}$ by a function that is linear in $p$,

$$
f_{\text {approx }}(x, p)=f\left(x, p_{0}\right)+\delta p F_{1}\left(x, p_{0}\right),
$$

where $F_{n}(x, p)=d^{n} f(x, p) / d p^{n}$. Equation (A2) is exact at $p_{0}: f_{\text {approx }}\left(x, p_{0}\right)=f\left(x, p_{0}\right)$. As in the two-fluid model that was discussed in the Introduction, $f_{\text {approx }}(x, p)$ has an exact isosbestic point at $\left(x^{\star}\left(p_{0}\right), p_{0}\right)$ because $F_{1}\left(x, p_{0}\right)$ vanishes there.

Next we determine the difference between the function $f(x, p)$ and the linear approximation $f_{\text {approx }}(x, p)$. For this purpose, we first expand $F_{1}(x, p)$ near $x^{\star}(p)$ for some fixed $p$ and obtain

$$
F_{1}(x, p)=F_{1}\left(x^{\star}(p), p\right)+\left.\sum_{n=1}^{\infty} \frac{\left[x-x^{\star}(p)\right]^{n}}{n !} \frac{\partial^{n} F_{1}}{\partial x^{n}}\right|_{x=x^{\star}(p)} .
$$

By virtue of the definition of $x^{\star}(p)$ [Eq. (1), the $x$-independent term vanishes, i.e., $F_{1}\left(x^{\star}(p), p\right)=0$, leading to

$$
F_{1}(x, p)=c(p)\left[x-x^{\star}(p)\right]+O\left\{\left[x-x^{\star}(p)\right]^{2}\right\},
$$

with $c(p)=\partial F_{1}(x, p) /\left.\partial x\right|_{x=x^{\star}(p)}$.

The height of the crossing region is thus given by

$$
\begin{aligned}
\delta f(x, p) & =f(x, p)-f_{\text {approx }}(x, p) \\
& =\frac{\delta p^{2}}{2} F_{2}\left(x, p_{0}\right)+\frac{\delta p^{3}}{6} F_{3}\left(x, p_{0}\right)+O\left(p^{4}\right) .
\end{aligned}
$$

Using Eq. (A4), we can now express the higher derivatives as $(n \geqslant 0)$

$$
\begin{aligned}
F_{n+1}(x, p)= & c^{(n)}(p)\left[x-x^{\star}(p)\right] \\
& -\sum_{k=1}^{n}\left(\begin{array}{l}
n \\
k
\end{array}\right) c^{(n-k)}(p) x^{\star(k)}(p),
\end{aligned}
$$

where $c^{(n)}(p)=\partial^{n} F_{1}(x, p) /\left.\partial^{n} x\right|_{x=x^{\star}(p)}$.

So far the parameter value $p$ has not been specified. We now suppose that $x^{\star}(p)$ has an extremum at $p_{0}$, in which case we term $\left(x^{\star}\left(p_{0}\right), p_{0}\right)$ a first-order isosbestic point. From $x^{\star^{\prime}}\left(p_{0}\right)=0$, it then follows that

$$
\delta f=\frac{c^{\prime}(p)}{2} \delta p^{2} \delta x+O\left(\delta p^{3} \delta x^{0}\right) .
$$

If instead $x^{\star}(p)$ has a saddle point at $p_{0}$ (second-order isosbestic point), then furthermore $x^{\star \prime}\left(p_{0}\right)=0$, yielding

$$
\delta f=\left(\frac{c^{\prime}(p)}{2} \delta p^{2}+\frac{c^{\prime \prime}(p)}{6} \delta p^{3}\right) \delta x+O\left(\delta p^{4} \delta x^{0}\right),
$$

and similar for higher-order isosbestic points. We thus conclude that an $n$ th-order isosbestic point, for which the first $n$ derivatives of $x^{\star}(p)$ vanish at $p_{0}$, will have a narrow crossing region,

$$
\begin{aligned}
\delta f & =\delta x \sum_{k=2}^{n+1} \frac{1}{k !} c^{(k-1)}(p) \delta p^{k}+O\left(\delta p^{n+2} \delta x^{0}\right), \\
& =O\left(\delta p^{n+2}\right) \quad \text { if } \quad \delta x=0,
\end{aligned}
$$

i.e., the first $n+1$ powers of $\delta p$ are suppressed by a factor $\delta x$, and the contribution of order $\delta x^{0}$ is suppressed by a factor $\delta p^{n+2}$. The last equation applies if we consider the height of the crossing region $\delta f\left(x^{\star}\left(p_{0}\right), p\right)$ for various parameters $p$ near $p_{0}$, and this height is only of order $O\left(\delta p^{n+2}\right)$ for an $n$ th-order crossing point. We conclude that the isosbestic point $x^{\star}(p)$ of a function $f(x, p)$ will be particularly sharp in the vicinity of an extremum or higher-order stationary point of $x^{\star}(p)$.

\section{APPENDIX B: QUANTIFICATION OF THE SHARPNESS OF ISOSBESTIC POINTS}

It is not possible to introduce an unambiguous measure for the sharpness of an approximate isosbestic point because 
of its explicit dependence on the magnification of the crossing region. This means that the observed sharpness always depends on the specifics of plotting. For fixed plot parameters, i.e., for $f(x, p)$ plotted for $x \in\left[x_{a}, x_{b}\right]$ and $p \in\left[p_{1}, p_{n}\right]$, one can, however, define a measure for the relative sharpness of a crossing region through the ratio between the area of the plot and the area of the crossing region. For this we define the vertical width of the plot as

$$
\Delta f=\max _{i, x_{1}, x_{2}}\left|f\left(x_{1}, p_{i}\right)-f\left(x_{2}, p_{i}\right)\right|
$$

with $i=1, \ldots, n$ and $x_{1,2} \in\left[x_{a}, x_{b}\right]$, and correspondingly the vertical width of the crossing region as

$$
\Delta f^{\star}=\max _{i, j}\left|f_{i}^{\star}-f_{j}^{\star}\right|,
$$

where $f_{i}^{\star}=f\left(x^{\star}\left(p_{\mathrm{i}}\right), p_{i}\right)$. With the horizontal width of the crossing region defined by

$$
\Delta x^{\star}=\max _{i, j}\left|x^{\star}\left(p_{i}\right)-x^{\star}\left(p_{j}\right)\right|,
$$

one can introduce a quantity $S$ through

$$
S=\left[\frac{\Delta f^{\star}}{\Delta f} \frac{\Delta x^{\star}}{\Delta x}\right]^{-1},
$$

where $\Delta x=\left|x_{a}-x_{b}\right|$, which quantifies the sharpness, i.e., $S=\infty$ for an exact isosbestic points and $S \gg 1$ for sufficiently sharp isosbestic points. We stress the explicit dependence of $S$ on $\Delta x$ and the range of parameters $\left[p_{1}, p_{n}\right]$ unless $S=\infty$. For finite $S$, the qualification "sharp" should thus be considered a relative term and be used with caution.

\section{APPENDIX C: EXISTENCE OF ISOSBESTIC POINTS IN $\sigma(\omega)$}

First we investigate the system-specific mechanisms that lead to isosbestic points in $\sigma(\omega, n)$. To allow for a rigorous analysis, we restrict ourselves only to cases in which there exists at most one isosbestic point. This applies to the results ${ }^{37}$ obtained for the Gaussian DOS as well as to our calculations for the semielliptic DOS. Due to particle-hole symmetry, one has $\sigma(\omega, \mu)=\sigma(\omega,-\boldsymbol{\mu})$. According to Eq. (1), isosbestic points are determined by

$$
\left.\frac{\partial \sigma\left(\omega^{\star}(n), n\right)}{\partial n}\right|_{\omega^{\star}(n)}=0 .
$$

In view of the one-to-one mapping between the chemical potential $\mu$ and the carrier density $n$ in the ungapped system, which is a direct consequence of the Green function $G(\omega, \mu)$ being a function only of $\omega+\mu$ (see Appendix E), this is equivalent to

$$
\left.\frac{\partial \sigma(\omega, \mu)}{\partial \mu}\right|_{\omega^{\star}(\mu)}=0 .
$$

Since $\Sigma(\omega)$ is also only a function of $\omega+\mu$ (see Appendix E), we obtain

$$
\begin{aligned}
\frac{\partial \sigma(\omega, \mu)}{\partial \mu}= & \sigma_{0} \int d \epsilon \rho(\epsilon) v(\epsilon)^{2} \int d \omega^{\prime} \frac{\partial}{\partial \omega^{\prime}} \\
& \times\left[A_{\epsilon}\left(\omega^{\prime}\right) A_{\epsilon}\left(\omega^{\prime}+\omega\right)\right] \frac{f\left(\omega^{\prime}\right)-f\left(\omega^{\prime}+\omega\right)}{\omega} .
\end{aligned}
$$

Integration by parts and using the property $\partial f(\omega) / \partial \omega=$ $-\delta(\omega)$ of the Fermi function gives

$$
\frac{\partial}{\partial \mu} \sigma(\omega, \mu)=\frac{\sigma_{0}}{\omega} \int_{-\infty}^{\infty} d \epsilon \tilde{\rho}(\epsilon) A_{\epsilon}(0)\left[A_{\epsilon}(\omega)-A_{\epsilon}(-\omega)\right]
$$

at $T=0$.

In Appendix D, we show that for less than half-filling, the derivative $\partial \sigma(\omega, \mu) / \partial \mu$ is positive around a $\omega_{0}>0$ and never becomes negative in the high-frequency limit. Since we assume the existence of at most one isosbestic point, it follows that it is exclusively determined by the $\mu$ dependence of the dc conductivity $\sigma_{\mathrm{dc}}(\mu)=\sigma(\omega=0, \mu)$ :

$$
\frac{\partial}{\partial \mu} \sigma_{\mathrm{dc}}(\mu)<0
$$

for less than half-filling. This relation expresses the equivalence between the existence of isosbestic points and the formation of a suppression of dc conductivity $\left[\sigma_{\mathrm{dc}}(\mu)\right.$ and $\sigma_{\mathrm{dc}}(n)$, respectively] around half-filling.

\section{APPENDIX D: HIGH-FREQUENCY LIMIT}

We determine the sign of $\partial \sigma\left(\omega_{0}\right) / \partial \mu$ in the high-frequency limit, taking $\mu<0$. In this case, the Green function and the self-energy are shifted to higher energies by $\mu$ compared to the half-filled case. For a symmetric DOS with finite bandwidth (Fig. 11), it follows from Eq. (C4) that there exists a frequency $\omega_{0}>0$ with $A\left(\omega_{0}\right)>0$ and $A\left(-\omega_{0}\right)=0$ (cf. Fig. 8). This yields $\partial \sigma\left(\omega_{0}\right) / \partial \mu>0$ because the contribution involving $A_{\epsilon}\left(-\omega_{0}\right)$ in Eq. (C4) must identically vanish due to the finite support of $\rho(\epsilon)$ and $\widetilde{\rho}(\epsilon)$. Symmetric densities of states with infinite bandwidth behave similarly: There exists an $\omega_{0}$ with $A(\omega)>A(-\omega)$ for all $\omega>\omega_{0}$ because of the stronger suppression of the contribution involving $A(-\omega)$. This analogously leads to $\partial \sigma\left(\omega_{0}\right) / \partial \mu>0$.

\section{APPENDIX E: SHAPE INVARIANCE OF SINGLE-PARTICLE QUANTITIES WITH RESPECT TO DOPING}

Within DMFT, the self-energy of the model can be expressed explicitly in terms of the interacting Green

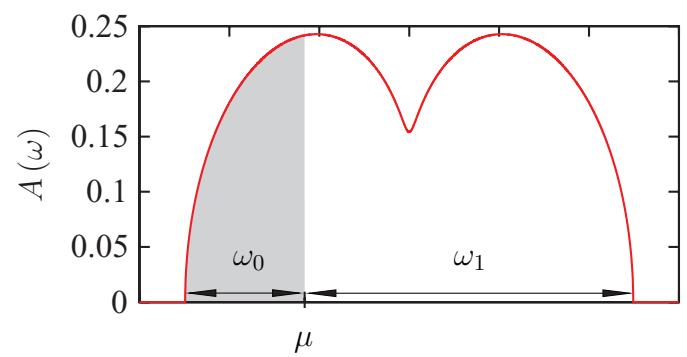

FIG. 11. (Color online) DOS with finite support $(\mu<0$, the shaded area indicates filled states): The term involving $A_{\epsilon}(-\omega)$ in Eq. (C4) vanishes for all frequencies $\omega>\omega_{0}$, while $A_{\epsilon}(\omega)>0$ for all $\omega_{0}<\omega<\omega_{1}$. Thus, the derivative $\partial \sigma\left(\omega_{0}\right) / \partial \mu$ will necessarily be positive for all $\omega_{0}<\omega<\omega_{1}$; for even higher frequencies it vanishes identically. 


$$
\begin{aligned}
& \text { function }^{37,57}\left(w_{0}=1-w_{1}\right): \\
& \Sigma(\omega)=\frac{U}{2}-\frac{1}{2}\left[\frac{1}{G(\omega)} \pm \sqrt{\left.\left(U-\frac{1}{G(\omega)}\right)^{2}+\frac{4 w_{0} U}{G(\omega)}\right]},\right.
\end{aligned}
$$

representing the exact summation of the skeleton expansion for $\Sigma(\omega)$. In the low- $U$ regime, an expansion of $\Sigma(\omega)$ in terms of the small parameter $U G(\omega)$ gives $\left(w_{1}=1 / 2\right)$

$$
\Sigma(\omega)=\frac{1}{2} U+\frac{1}{4} U^{2} G(\omega)-\frac{1}{16} U^{4} G^{3}(\omega)+O\left[U^{6}\right]
$$

representing the first few terms of its skeleton expansion. As a consequence of Eq. (26), the model has an especially simple doping dependence, where a change of $\mu$ (for constant $w_{1}$ ) merely shifts the Green function and the self-energy, leaving their shape invariant. Mathematically, this is expressed by the fact that $\omega$ and $\mu$ enter $G(\omega)$ and $\Sigma(\omega)$ only through $\omega+\mu$. To see that, we consider the inverse ${ }^{46}$ of the Hilbert transform $R[G(\omega)]$, expressing $\omega+\mu-\Sigma(\omega)$ exclusively through $G(\omega)$. Equation (26) then takes the form

$$
\begin{aligned}
G(\omega)= & \frac{1-w_{1}}{\omega+\mu-R[G(\omega)]+G^{-1}(\omega)} \\
& +\frac{w_{1}}{\omega+\mu-R[G(\omega)]+G^{-1}(\omega)-U},
\end{aligned}
$$

implicitly defining the Green function as a function of $\omega+\mu$, i.e., $G(\omega)=F(\omega+\mu)$. For the special case of a semielliptic DOS, Eq. (E3) leads to a cubic equation, which can be solved explicitly $^{60}$ for $G(\omega+\mu)$.
${ }^{1}$ G. Scheibe, Ang. Chem. 50, 212 (1937).

${ }^{2}$ M. D. Cohen and E. Fischer, J. Chem. Soc. 3044 (1962).

${ }^{3}$ J. R. Morrey, J. Phys. Chem. 67, 1569 (1963).

${ }^{4}$ Isosbestic: from the Greek isos ("equal") + sbestos (derived from sbennynai "to quench" or "to extinguish").

${ }^{5}$ R. K. Poole and I. U. Kalnenieks, in Spectrophotometry and Spectrofluorimetry: A Practical Approach, edited by M. Gore (Oxford University Press, New York, 2000).

${ }^{6}$ S. Uchida, T. Ido, H. Takagi, T. Arima, Y. Tokura, and S. Tajima, Phys. Rev. B 43, 7942 (1991).

${ }^{7}$ J. K. Freericks, T. P. Devereaux, and R. Bulla, Phys. Rev. B 64, 233114 (2001).

${ }^{8}$ D. S. Greywall, Phys. Rev. B 27, 2747 (1983).

${ }^{9}$ D. Vollhardt, Phys. Rev. Lett. 78, 1307 (1997).

${ }^{10}$ G. E. Brodale, R. A. Fisher, N. E. Phillips, and J. Flouquet, Phys. Rev. Lett. 56, 390 (1986).

${ }^{11}$ H. G. Schlager, A. Schröder, M. Welsch, and H. Löhneysen, J. Low Temp. Phys. 90, 181 (1993).

${ }^{12}$ B. Belevtsev, V. Krasovitsky, D. Naugle, K. Rathnayaka, G. Agnolet, and I. Felner, J. Phys.: Condens. Matter 21, 455602 (2009).

${ }^{13}$ A. Bauer, A. Neubauer, C. Franz, W. Münzer, M. Garst, and C. Pfleiderer, Phys. Rev. B 82, 064404 (2010).

${ }^{14}$ M. Janoschek, M. Garst, A. Bauer, P. Krautscheid, R. Georgii, P. Böni, and C. Pfleiderer, Phys. Rev. B 87, 134407 (2013).

${ }^{15}$ T. Adams, A. Chacon, M. Wagner, A. Bauer, G. Brandl, B. Pedersen, H. Berger, P. Lemmens, and C. Pfleiderer, Phys. Rev. Lett. 108, 237204 (2012).

${ }^{16}$ K. Seiler, C. Gros, T. M. Rice, K. Ueda, and D. Vollhardt, J. Low Temp. Phys. 64, 195 (1986).

${ }^{17}$ A. Georges and W. Krauth, Phys. Rev. B 48, 7167 (1993).

${ }^{18}$ N. Chandra, M. Kollar, and D. Vollhardt, Phys. Rev. B 59, 10541 (1999).

${ }^{19}$ H. Shiba and P. A. Pincus, Phys. Rev. B 5, 1966 (1972).

${ }^{20}$ H. Shiba, Prog. Theor. Phys. 48, 2171 (1972).

${ }^{21}$ G. Jüttner, A. Klümper, and J. Suzuki, Nucl. Phys. B 522, 471 (1998).

${ }^{22}$ D. Duffy and A. Moreo, Phys. Rev. B 55, 12918 (1997).

${ }^{23}$ T. Paiva, R. T. Scalettar, C. Huscroft, and A. K. McMahan, Phys. Rev. B 63, 125116 (2001).

${ }^{24}$ C. A. Macedo and A. M. C. de Souza, Phys. Rev. B 65, 153109 (2002).
${ }^{25}$ T. Paiva, R. T. Scalettar, W. Zheng, R. R. P. Singh, and J. Oitmaa, Phys. Rev. B 72, 085123 (2005).

${ }^{26}$ F. Gebhard and A. E. Ruckenstein, Phys. Rev. Lett. 68, 244 (1992).

${ }^{27}$ The fact that the high-temperature crossing point in $c(T, U)$ for the one-band Hubbard model occurs at a nearly universal value $c^{*} / k_{B} \simeq 0.34$ can be shown to be a consequence of the existence of two small parameters: the integral over the deviation of the density of states from a constant value, and the inverse dimension, $1 / d .^{18}$

${ }^{28}$ S. Glocke, A. Klümper, and J. Sirker, Phys. Rev. B 76, 155121 (2007).

${ }^{29}$ T. A. Costi and V. Zlatić, Phys. Rev. B 81, 235127 (2010).

${ }^{30}$ M. Brando, L. Pedrero, T. Westerkamp, C. Krellner, P. Gegenwart, C. Geibel, and F. Steglich, Phys. Status Solidi B 250, 485 (2013).

${ }^{31}$ M. Imada, A. Fujimori, and Y. Tokura, Rev. Mod. Phys. 70, 1039 (1998).

${ }^{32}$ I. Kézsmárki, N. Hanasaki, K. Watanabe, S. Iguchi, Y. Taguchi, S. Miyasaka, and Y. Tokura, Phys. Rev. B 73, 125122 (2006).

${ }^{33}$ W. Guyard, M. Le Tacon, M. Cazayous, A. Sacuto, A. Georges, D. Colson, and A. Forget, Phys. Rev. B 77, 024524 (2008).

${ }^{34}$ N. N. Kovaleva, A. V. Boris, C. Bernhard, A. Kulakov, A. Pimenov, A. M. Balbashov, G. Khaliullin, and B. Keimer, Phys. Rev. Lett. 93, 147204 (2004)

${ }^{35}$ C. Kant, T. Rudolf, F. Mayr, S. Krohns, P. Lunkenheimer, S. G. Ebbinghaus, and A. Loidl, Phys. Rev. B 77, 045131 (2008).

${ }^{36}$ J. K. Freericks, T. P. Devereaux, R. Bulla, and T. Pruschke, Phys. Rev. B 67, 155102 (2003).

${ }^{37}$ J. K. Freericks and V. Zlatić, Rev. Mod. Phys. 75, 1333 (2003).

${ }^{38}$ A. M. Shvaika, O. Vorobyov, J. K. Freericks, and T. P. Devereaux, Phys. Rev. Lett. 93, 137402 (2004).

${ }^{39}$ M. Eckstein, M. Kollar, and D. Vollhardt, J. Low Temp. Phys. 147, 279 (2007).

${ }^{40}$ Of course, this width depends on the linear scale of the axes: only if the curves cross in one mathematically defined point is the linear scale irrelevant; see the discussion in Sec. II B and Appendix B.

${ }^{41}$ In Refs. $13-15$, the crossing point observed in the specific-heat curves $C(H, T)$, i.e., the near independence of $C(H, T)$ on the magnetic field $H$ at some temperature $T^{\star}$, is referred to as "Vollhardt invariance."

${ }^{42}$ W. Metzner and D. Vollhardt, Phys. Rev. Lett. 62, 324 (1989).

${ }^{43}$ A. Georges and G. Kotliar, Phys. Rev. B 45, 6479 (1992).

${ }^{44}$ D. Vollhardt, in Correlated Electron System, edited V. J. Emery (World Scientific, Singapore, 1993). 
${ }^{45}$ T. Pruschke, M. Jarrell, and J. Freericks, Adv. Phys. 44, 187 (1995).

${ }^{46}$ A. Georges, G. Kotliar, W. Krauth, and M. J. Rozenberg, Rev. Mod. Phys. 68, 13 (1996).

${ }^{47}$ G. Kotliar and D. Vollhardt, Phys. Today 57(3), 53 (2004).

${ }^{48}$ More generally, this applies in particular also to any kind of two-fluid model employed, for example, in phenomenological theories of superconductivity and superfluidity. There the density of the two components (e.g., the normal and superfluid component) depend on temperature while the total density is constant: $n=$ $n_{1}(T)+n_{2}(T)=$ const. The properties of the system are then described by the superposition of the two components, leading to a special dependence of quantities $f(T, X)$ on $T$ and $X$ of the form $f(T, X)=n_{1}(T) f_{1}(X)+\left[n-n_{1}(T)\right] f_{2}(X)$. This implies the crossing of curves for different temperatures $T$ at a single point $X^{*}$ determined by $f_{1}\left(X^{*}\right)=f_{2}\left(X^{*}\right)$.

${ }^{49}$ I. Kézsmárki (private communication).

${ }^{50}$ Other combinations (e.g., $T_{1}=40 \mathrm{~K}, T_{2}=80 \mathrm{~K}$, etc.) showed no significantly different behavior of $\tilde{\chi}(\omega, T)$ and $\chi_{2}(\omega)$, apart from an eventual increase in noise in $\chi_{2}(\omega)$, which can become larger than the numerator in the expression for $\chi_{2}(\omega)$ for combinations such as $T_{1}=14 \mathrm{~K}$ and $T_{2}=20 \mathrm{~K}$.

${ }^{51}$ K. Okazaki, H. Wadati, A. Fujimori, M. Onoda, Y. Muraoka, and Z. Hiroi, Phys. Rev. B 69, 165104 (2004).
${ }^{52}$ C. K. Chiang, C. R. Fincher, Y. W. Park, A. J. Heeger, H. Shirakawa, E. J. Louis, S. C. Gau, and A. G. MacDiarmid, Phys. Rev. Lett. 39, 1098 (1977).

${ }^{53}$ H. Shirakawa, E. J. Louis, A. G. MacDiarmid, C. K. Chiang, and A. J. Heeger, J. Chem. Soc. Chem. Commun. 578 (1977).

${ }^{54}$ C. A. Thomas, Ph.D. thesis, University of Florida, 2002.

${ }^{55}$ N. N. Kovaleva, A. M. Oleś, A. M. Balbashov, A. Maljuk, D. N. Argyriou, G. Khaliullin, and B. Keimer, Phys. Rev. B 81, 235130 (2010).

${ }^{56}$ L. M. Falicov and J. C. Kimball, Phys. Rev. Lett. 22, 997 (1969).

${ }^{57}$ U. Brandt and C. Mielsch, Z. Phys. B 75, 365 (1989).

${ }^{58}$ M. Jarrell, J. K. Freericks, and T. Pruschke, Phys. Rev. B 51, 11704 (1995).

${ }^{59}$ N. Blümer and P. G. J. van Dongen, in Concepts in Electron Correlation, Vol. 110 of NATO Science Series, edited by A. C. Hewson and V. Zlatić (Kluwer, Dordrecht, 2003).

${ }^{60}$ P. G. J. van Dongen, Phys. Rev. B 45, 2267 (1992).

${ }^{61}$ H. Schweitzer and G. Czycholl, Z. Phys. B 83, 93 (1991).

${ }^{62}$ R. Bulla, T. A. Costi, and T. Pruschke, Rev. Mod. Phys. 80, 395 (2008).

${ }^{63}$ Here we used the NRG LJUBLJANA package by R. Žitko, http://auger.ijs.si/ljubljana/; R. Žitko, Comput. Phys. Commun. 182, 2259 (2011). 